\section{Surgical pearl on reconstructing surgical defects of the nasal tip}

\author{
Artur César, ${ }^{1,2}$ Ana Barros, ${ }^{1}$ \\ Paulo Santos, ${ }^{1}$ Filomena Azevedo ${ }^{1}$ \\ 'Department of Dermatology and \\ Venereology, Centro Hospitalar São João \\ EPE, Porto; ' ${ }^{\text {Faculty }}$ of Medicine, \\ University of Porto, Portugal
}

\section{Abstract}

Repairing surgical defects of the nasal tip is challenging, mainly because of the lack of freely mobile skin available peripherally. The Peng flap is a one-stage cutaneous flap that circumvents this difficulty by recruiting skin from the nasal dorsum and sidewall regions. The design produces a tridimensional shape perfectly adapted to the configuration of the nasal tip and allows for an inconspicuous closure of the defect. We present three examples of full-thickness skin defects involving the nasal tip, reconstructed using a modified version of the Peng flap, and present the experience at our department with this surgical technique.

\section{Introduction}

Basal cell carcinoma (BCC) is the most common non-melanoma skin cancer of the head and neck regions. ${ }^{1}$ About $80 \%$ of all BCCs occur on the face and $30 \%$ on the nose. ${ }^{2}$ Surgical excision is the gold-standard treatment, however, closing the resulting surgical defects on the nasal tip is often difficult, mainly because of the lack of available freely mobile skin peripherally.

\section{Case Report}

Herein we present three cases in which a modified version of a Peng flap was used to reconstruct skin defects following excision of BCCs on the nasal tip (one nodular, one micronodular and one morpheaform). Surgical excisions were performed with a $4-5 \mathrm{~mm}$ of clinically normal surrounding tissue. The resulting full-thickness skin defects measured between $1.6-2.0 \mathrm{~cm}$ in the horizontal plane and $1.6-2.2 \mathrm{~cm}$ in the vertical plane (Figure 1A-C). Wound closures were performed two weeks following the excision, after obtaining histopathology confirmation of tumor-free peripheral and deep margins. The design and intraoperative steps of the employed modified version of the Peng flap are represented in Figure 2.

Under local anesthesia (with 1\% lidocaine), the incisions are started at the distal margins of the defect, continuing laterally and superiorly, along the alar grooves and the junction between the nasal sidewalls and cheeks.

Once the skin is incised, the flap and the surrounding skin are undermined in the submuscular and subcutaneous plane, respectively. At this point, a meticulous hemostasis must be ensured using thermal cautery to prevent any hematoma that could compromise the flap's survival.

The distal tips of each of the arms of the flap may need to be trimmed to the appropriate thickness before being sutured in place using 4-0 deep absorbable sutures.

Subsequently, the standing cone at the center of both arms of the flap is removed. In midline defects this standing cone should be directed vertically, whereas in eccentric defects the cone must directed towards the wider of the two arms of the flap.

Once the deep absorbable sutures are placed along all aspects of the flap, removal of standing tissue cones at the medial canthi is sometimes necessary to prevent dog-ear deformities.

Finally, the epidermis is approximated using a 5-0 non-absorbable suture with appropriate evertion of the wound edges.

The non-absorbable sutures are removed between eight to ten days following the procedure and the patients are reevaluated monthly thereafter for the first four months.

\section{Results}

The postoperative recovery was uneventful. Figure 1D-F shows the cosmetic results four months after the reconstructive surgery without visible distortion of the nasal anatomy and with very good cosmetic outcomes, even this early on.

\section{Discussion}

Repairing surgical defects of the nasal tip has particular difficulties given its tridimensional shape and limited area from which to recruit similar skin (in terms of skin thickness, color and adnexal composition). The most frequently employed closure methods are primary closure, skin flaps and skin grafts.

The Peng flap is an advancement-rotation cutaneous flap first described by Peng et al, in $1987^{3}$ The concept behind the Peng flap is a pinch modification of the Rintala flap (glabel-
Correspondence: Artur Jorge Fernandes César, Department of Dermatology and Venereology, Centro Hospitalar São João EPE, Alameda Prof. Hernani Monteiro, 4200-319 Porto, Portugal.

Tel.: +35.122 .551 .2117$

E-mail: arturjfc@gmail.com

Key words: Surgical flaps; Nose.

Contributions: the authors contributed equally.

Conflict of interest: the authors declare no potential conflict of interest.

Received for publication: 9 August 2016.

Accepted for publication: 7 November 2016.

This work is licensed under a Creative Commons Attribution-NonCommercial 4.0 International License (CC BY-NC 4.0).

(C) Copyright A. César et al., 2016

Licensee PAGEPress, Italy

Dermatology Reports 2016; 8:6828

doi:10.4081/dr.2016.6828

lar linear advancement flap), classically used to repair defects of the dorsum and nasal tip. This modification allows the closure of larger defects on the nasal tip by taking advantage from the nasal sidewall skin excess bilaterally and improves flap survival by requiring shorter pedicles. Furthermore, it produces a convex configuration perfectly adapted to the shape of the nasal tip and recruits skin of color and texture very similar to the perilesional area. ${ }^{4}$

In 1995, Rowe et al proposed initiating the incisions for the rotating arms at the most distal portion of the defect, reducing the advancement movement of the flap. ${ }^{4}$ Recently, Ahern et $a l$, added an additional modification by placing the incisions more laterally along the alar grooves and the junction between the cheek and nasal sidewall. On the vast majority of nasal full-thickness cutaneous defects of the nasal tip we encounter at our department, we perform the latest modified version of Peng flap. This has to do with the larger pedicle base improving flap survival, but also because it produces better cosmetic outcomes since the incisions are placed along the junction between adjacent aesthetic subunits. ${ }^{5}$ Moreover, the unique symmetric design of this flap minimizes the risk of asymmetric distortion of the nose, particularly of the nasal alae, a complication occasionally observed with other flap designs used on the nasal tip. ${ }^{4}$

In our experience of more than 20 patients subjected to this procedure, the Peng flap produces excellent results in medium/large nasal tip defects, centrally or eccentrically located, as well as defects that bridge the distal nasal $d o r$ sum and nasal tip. However, we agree with other authors that this flap is not suitable for most defects covering more than $50 \%$ of the 
nasal tip subunit or deep defects involving the cartilage. In these cases, the additional bulk of a paramedian forehead flap, or composite skin

grafts, may be preferable. We have not observed any case of distortion or major complication with this technique, nonetheless this

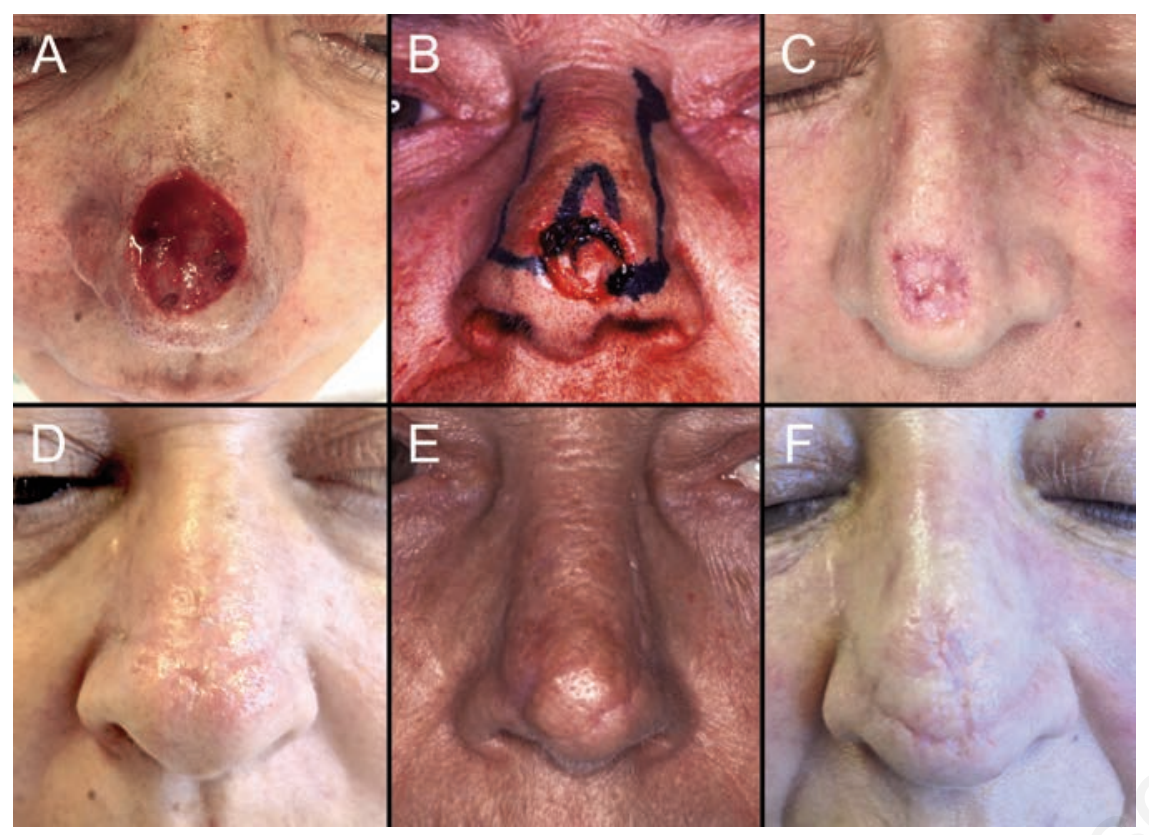

Figure 1. A, B and C) Surgical defects two weeks following excision of basal cell carcinoma on the nose. Photographs $A$ and $B$ were taken after removing the scar tissue formed by secondary intention healing during this two-week interval. Photograph $\mathrm{C}$ was taken before removal of the scar tissue. D, E and F) Results four months after the surgery.

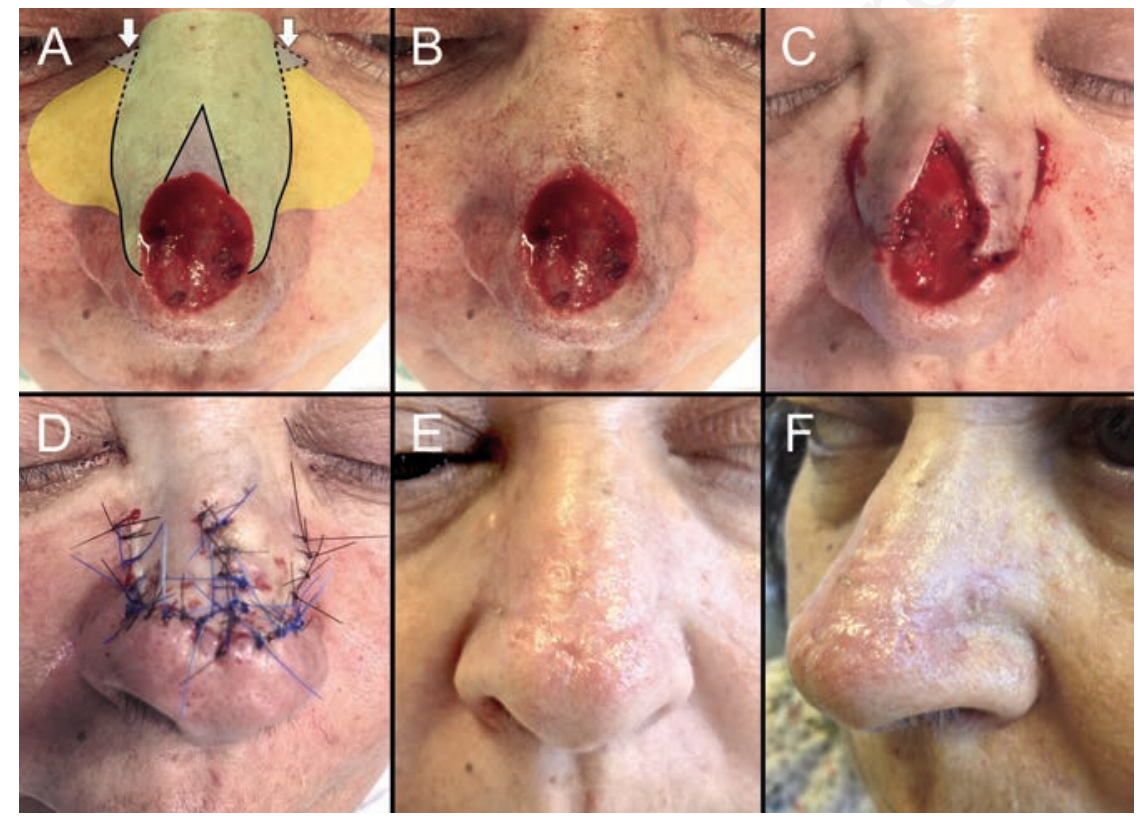

Figure 2. A) Flap design: the incisions (continuous lines) are started at the distal margins of the defect and continued laterally and superiorly; to facilitate the advancement movement the incisions can be extended up to the medial canthal region (interrupted lines); undermining of the flap in the submuscular plane (green) and surrounding regions in the subcutaneous plane (yellow) must be performed; a $30^{\circ}$ central standing cone is removed and Burrow's triangles in the superior region of the flap may be excised if needed (white arrows); B) Surgical defect following excision of a nodular BCC on the nasal tip extending to the distal dorsum of the nose; C) Intraoperative view of flap prepared for final closure; D) Postoperative view of completed reconstruction; E and F) Results four months after the surgery. flap is not without possible complications. Cases of partial flap necrosis and infection, elevation of the nasal alae in larger defects, as well as asymmetries in eccentric defects have been described. ${ }^{4,5}$

\section{Conclusions}

The modified Peng flap is a one-stage reconstruction procedure that enables the closure of defects of the nasal tip of medium-large sizes, extending to, or adjacent to the nasal dorsum.

This flap generally produces an excellent skin color and texture match and preserves the nasal architecture. As a result, it is the authors' opinion that it is an essential tool to have in the armamentarium of dermatological surgeons for reconstructing nasal tip defects.

\section{References}

1. McGuire JF, Ge NN, Dyson S. Nonmelanoma skin cancer of the head and neck I: histopathology and clinical behavior. Am J Otolaryngol 2009;30:121-33.

2. Choi JH, Kim YJ, Kim H, et al. Distribution of basal cell carcinoma and squamous cell carcinoma by facial esthetic unit. Arch Plast Surg 2013;40:387-91.

3. Peng VT, Sturm RL, Marsh TW. Pinch modification of the linear advancement flap. J Dermatol Surg Oncol 1987;13:251-3.

4. Rowe D, Warshawski L, Carruthers A. The Peng flap. The flap of choice for the convex curve of the central nasal tip. Dermatol Surg 1995;21:149-52.

5. Ahern RW, Lawrence N. The Peng flap: reviewed and refined. Dermatol Surg 2008;34:232-7. 